Jacek Kubica, Zbigniew Włodarczyk, Wioleta Stolarek, Emilia Wojtal, Katarzyna Buszko, Klaudyna Grzelakowska, Agata Kosobucka-Ozdoba, Magdalena Krintus, Jacek Kryś, Aldona Kubica, Piotr Lackowski, Piotr Michalski, Maciej Piasecki, Eliano P. Navarese

\title{
Determinants of the level of anti-SARS- CoV-2 IgG ANTibodiEs after vaccination (DANTE-SIRIO 7) study. A rationale and protocol of the study
}

\section{Corresponding author:}

Wioleta Stolarek, Collegium Medicum, Nicolaus Copernicus University, Marii Skłodowskiej-Curie 9 St. 85-094 Bydgoszcz, Poland, e-mail: wioletaplazuk@o2.pl
Medical Research Journal 2021; Volume 6. Number 4, 312-315 10.5603/MRJ.a2021.0052 Copyright @ 2021 Via Medica ISSN 2451-2591 e-ISSN 2451-4101

\begin{abstract}
Introduction: Great expectations to control the pandemic are placed on vaccines against COVID-19. So far, four COVID-19 vaccines have been approved for use in the European Union. This study aimed to assess the anti-SARS-CoV-2 IgG antibody concentration after the vaccination cycle with the BNT162b2 vaccine at several predefined time points. All results will be compared to the COVID-19 history and severity of symptoms during the disease and after the first and second vaccine dose.

Material and methods: The study includes healthy, unselected volunteers from the staff of Dr. Antoni Jurasz University Hospital No.1 in Bydgoszcz and students of the Collegium Medicum, Nicolaus Copernicus University. To meet the inclusion criteria all individuals will have to be vaccinated with two doses of the BNT162b2 (BioNTech/Pfizer, US) vaccine. Anti-SARS-CoV-2 IgG antibody concentrations will be measured in fresh serum samples collected from each participant at predefined time points. All measurements will be performed using the Atellica system (Siemens Healthineers, Erlangen, Germany). Results of SARS-CoV-2 IgG are given as $\mathrm{U} / \mathrm{mL}$, whereby the cut-off for positivity is defined as $\geq 1.0 \mathrm{U} / \mathrm{mL}$.

Discussion: This study was designed to evaluate the impact of COVID-19 on the severity of clinical symptoms and the levels of anti-SARS-CoV-2 IgG antibodies after vaccination. Hopefully, based on its results, further clinical outcome-powered trials will be designed and conducted to elucidate how quantitative antibody levels can be used as a correlate of vaccine-mediated protection.

Key words: SARS-CoV-2; COVID- 19, anti-SARS-CoV-2 IgG antibodies; COVID-19 vaccine
\end{abstract}

Med Res J 2021; 6 (4): 312-315

\section{Introduction}

The coronavirus disease 2019 (COVID19) outbreak caused by the severe acute respiratory syndrome coronavirus 2 (SARS-CoV-2) is one of the most devastating worldwide crises in recent years. Individuals can be protected from SARS-CoV-2 via immunity that is induced by either a previous SARS-CoV-2 infection or vaccination [1]. Great expectations to control the pandemic are placed on vaccines against COVID-19. So far, four COVID-19 vaccines approved in the European Union are the messenger RNA (mRNA)-based BNT162b2 and mRNA-1273, and the adenoviral vector-based ChAdOx1 nCoV-19 and Ad26.COV2-S [2]. All these vaccines were designed to elicit an immune response directed toward the S1 spike protein of SARS-CoV-2 [3]. Both BNT162b2 and mRNA-1273, within the first 100 days after vaccination, were able to elicit neutralizing antibodies concentrations and specific antibodies titers higher than those observed in the COVID-19 human convalescent serum [4]. The SARS-CoV-2 mRNA vaccines, i.e. BNT162b2 and mRNA-1273 (Moderna), have been shown to have more than $90 \%$ efficacy in preventing COVID-19 illness [5, 6]. The efficacy of the BNT162b2 vaccines against symptomatic laboratory-confirmed SARS-CoV-2 infection has been reported in a large randomized controlled clinical trial as 52\% (95\% confidence interval $(\mathrm{Cl})=30-86 \%)$ after the first dose and 
95\% (95\% Cl=90-98\%) after the second dose of the vaccine [6]. Interestingly, antibody concentrations in the vaccinated candidates decreased by up to $40 \%$ in the subsequent months. However, such a rapid decrease was not observed in the case of convalescent subjects, where the antibody titer decreased by less than $5 \%$ per month [7]. Successive scientific reports enrich knowledge about the organism's response to anti-SARS-CoV-2 vaccination; however, still many questions regarding the determinants of antibody levels remain unanswered. Therefore, we have designed the study aimed to assess the anti-SARSCoV-2 IgG antibody concentration after the vaccination cycle with the BNT162b2 vaccine at several time points and relate these results to the COVID-19 history and severity of symptoms during the disease and after the first and second vaccine dose.

\section{Material and methods}

The study was designed as a prospective, single-center, longitudinal observational study that includes healthy, unselected volunteers from the staff of Dr. Antoni Jurasz University Hospital No.1 in Byd- goszcz and students of Collegium Medicum, Nicolaus Copernicus University (Poland). The study protocol was approved by the Ethics Committee of the Nicolaus Copernicus University in Torun functioning at Collegium Medicum in Bydgoszcz (approval number KB 160/2021). The study was registered in the ClinicalTrials. gov database and received identifier NCT05109585.

Approximately 1000 adult participants are planned to be included in the study. To meet the inclusion criteria subjects will have to be vaccinated with two doses of the BNT162b2 vaccine. All individuals will be examined at four time points: $3,6,9$, and 12 months after the second dose of vaccine (the DANTE-SIRIO 7). The study protocol was modified according to the new guidelines regarding a third dose of the vaccine against COVID-19 in Poland. In all participants, who declare willingness to receive the third dose of the vaccine, anti-SARS-CoV-2 IgG antibody concentrations will be examined up to 7 days before administration of the third dose, then one and three months after the vaccination. In individuals not receiving the third dose of the vaccine, the anti-SARS-CoV-2 IgG antibodies will be measured according to the original schedule, i.e. at 9 and 12 months after the second dose of the vaccine (Fig. 1). Vaccination data will be verified

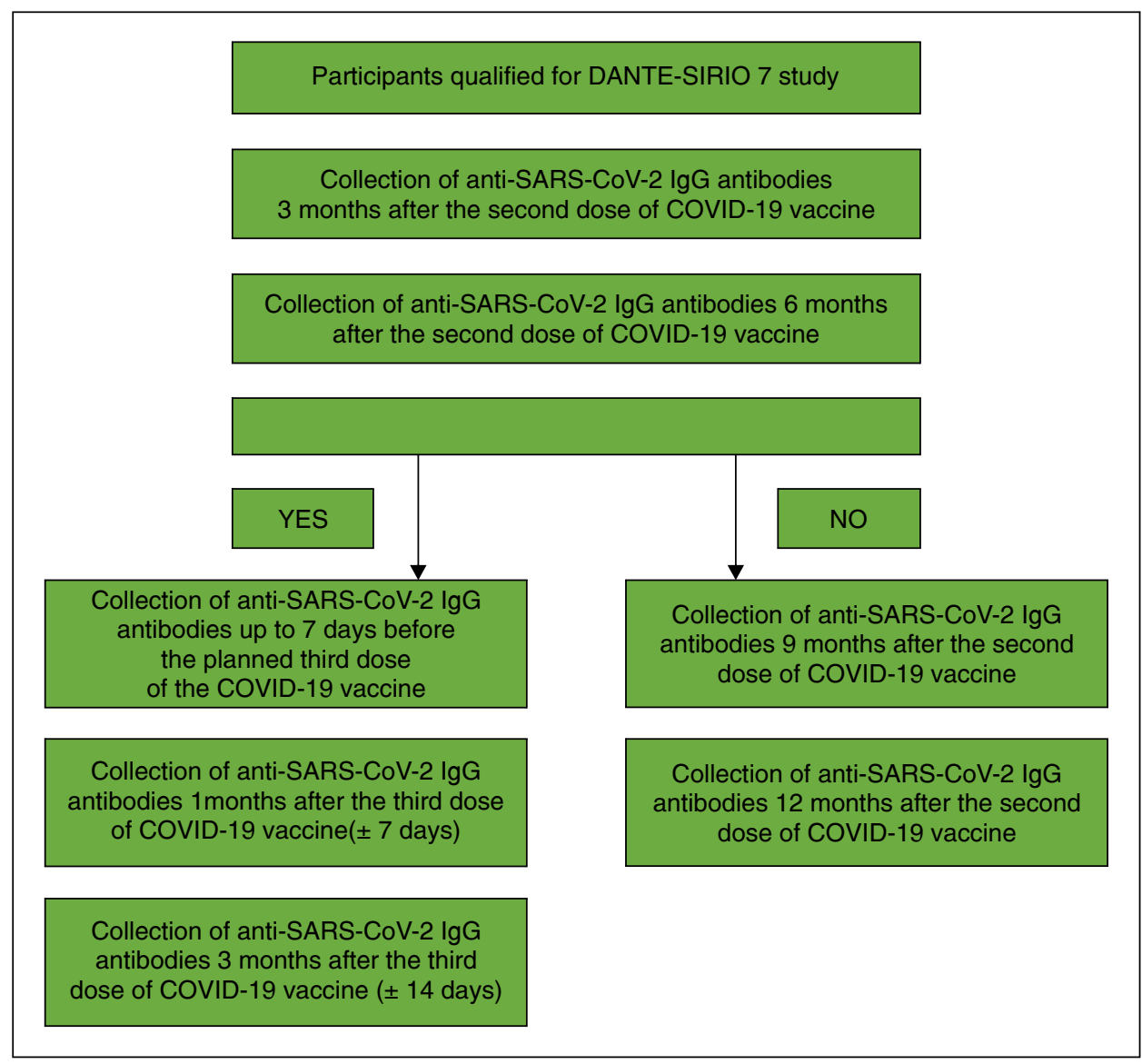

Figure 1. The schematic protocol of the study 
in the Dr. Antoni Jurasz University Hospital vaccination register center. Provision of informed consent will be mandatory to participate in the study. At the first time-point (3 months after vaccination), participants will be asked to fulfill a survey on sociodemographic characteristics, previous SARS-CoV-2 infection and accompanying symptoms, and symptoms after a dose of vaccination (7 questions in total). Moreover, possible SARS-CoV-2 infection will be assessed in each phase of the study with the use of an additional survey.

Fresh serum samples will be used to measure SARS-CoV-2 IgG concentrations on the Siemens Atellica system (Siemens Healthineers, Erlangen, Germany). The Atellica IM SCOVG assay is a fully automated 2step-sandwich immunoassay using acridinium-ester chemiluminescent technology. All samples will be processed according to the manufacturer's procedures with the specified controls and calibrators by trained laboratory staff. Results of SARS-CoV-2 IgG are given as $U / \mathrm{mL}$, whereby the cut-off for positivity is defined as $\geq 1.0 \mathrm{U} / \mathrm{mL}$. The manufacturer reports a range of quantification of 0.5 to $150.0 \mathrm{U} / \mathrm{mL}$, which may be extended to $750.0 \mathrm{U} / \mathrm{mL}$ upon automated 1:5 predilution. The lower limit of the analytical measuring interval corresponds to $0.50 \mathrm{U} / \mathrm{mL}$. The manufacturer reported a specificity of $99.90 \%$ (95\% Cl 99.64-99.99) and a sensitivity of $96.41 \%(95 \% \mathrm{Cl} 92.74$ to 98.44$)$ for samples $\geq 21$ days post RT-PCR confirmation. To achieve assay traceability to WHO 20/136 (BAU/mL), all reported results should be multiplied by 21.8 .

Primary endpoint:

- the anti-SARS-CoV-2 IgG antibody concentration 3 months after the vaccination cycle with the BNT162b2 vaccine.

Secondary endpoints:

- the anti-SARS-CoV-2 IgG antibody concentration 6, 9 , and 12 months after two-dose vaccination cycle;

- the anti-SARS-CoV-2 IgG antibody concentration immediately before, 1 and 3 months after the third vaccine dose;

- local and/or systemic adverse reactions after vaccination;

- confirmed COVID-19 after vaccination.

\section{Discussion}

The study will evaluate the anti-SARS-CoV-2 IgG antibody concentrations in a cohort of unselected healthy adult volunteers vaccinated with BNT162b2. This study was designed to evaluate the impact of COVID-19 on the severity of clinical symptoms and the levels of anti-SARS-CoV-2 IgG antibodies after vaccination. The strength of this study lies in the assessment of the anti-SARS-CoV-2 antibody concentrations at several time points in each participant. Hopefully, based on its results, further clinical outcome-powered trials will be designed and conducted to elucidate how quantitative antibody levels can be used as a correlate of vaccine-mediated protection. It is worth mentioning that the Department of Cardiology, Dr. A. Jurasz University Hospital No. 1 in Bydgoszcz, is conducting the ReCOVery-SIRIO Clinical Trial (clinicaltrials. gov ID: NCT04351763) aiming to evaluate the effect of drugs commonly used in cardiology, amiodarone and verapamil, on the clinical course of COVID-19. The study has ended and the results are under development.

It should be noted that the study will include healthcare workers, a group significantly affected by the COVID-19 pandemic, which can be observed through higher rates of infections and quarantines, especially among nurses and doctors. The COVID-19 pandemic has influenced the stability and availability of the workforce in the hospital both directly (infections, quarantines) and indirectly (sick leaves) [8]. It resulted in functioning changes and an increase of in-hospital mortality in the Department of Cardiology, Dr. A. Jurasz University Hospital No. 1 in Bydgoszcz. The observed decrease in the number of hospital admissions and specialized cardiac procedures performed in 2020 may have a serious impact on future patients' profiles [9]. In addition, as high prevalence of concomitant diseases is noted in patients with severe or fatal cases of SARS-CoV-2 infection, it is important to highlight that COVID-19 and type 1 diabetes may constitute a deadly duo characterized by mortality rates that are higher than in the non-diabetic population [10].

\section{Conflict of interest: None.}

\section{Funding: The study is funded by Collegium} Medicum of Nicolaus Copernicus University (NCU CM grant no. ZES. WL. 9.2021) and did not receive any external funding.

\section{References}

1. Tsai SC, Lu CC, Bau DT, et al. Approaches towards fighting the COVID19 pandemic (Review). Int J Mol Med. 2021; 47(1): 3-22, doi: 10.3892/ijmm.2020.4794, indexed in Pubmed: 33236131

2. COVID-19 vaccines authorised for use in the European Union. https:// www.ema.europa.eu/en/human-regulatory/overview/public-healththreats/coronavirus-disease-covid-19/treatments-vaccines/covid19-vaccines.

3. Lumley SF, O'Donnell D, Stoesser NE, et al. Oxford University Hospitals Staff Testing Group. Antibody status and incidence of SARS-CoV-2 infection in health care workers. N Engl J Med. 2021; 384(6): 533-540, doi: 10.1056/NEJMoa2034545, indexed in Pubmed: 33369366.

4. Lombardi A, Bozzi G, Ungaro R, et al. Mini review immunological consequences of immunization with COVID-19 mRNA vaccines: preliminary results. Front Immunol. 2021; 12: 657711, doi: 10.3389/fimmu.2021.657711, indexed in Pubmed: 33777055. 
5. Baden LR, El Sahly HM, Essink B, et al. COVE Study Group. Efficacy and safety of the mRNA-1273SARS-CoV-2 vaccine. NEngl J Med. 2021; 384(5): 403-416, doi: 10.1056/NEJMoa2035389, indexed in Pubmed: 33378609.

6. Polack FP, Thomas SJ, Kitchin N, et al. C4591001 Clinical Trial Group. Safety and efficacy of the BNT162b2 mRNA Covid-19 vaccine. N Engl J Med. 2020; 383(27): 2603-2615, doi: 10.1056/NEJMoa2034577, indexed in Pubmed: 33301246.

7. Lombardi A, Consonni D, Oggioni M, et al. SARS-CoV-2 anti-spike antibody titres after vaccination with BNT162b2 in naïve and previously infected individuals. J Infect Public Health. 2021; 14(8): 1120-1122, doi: 10.1016/i.jiph.2021.07.005, indexed in Pubmed: 34293641.
8. Grzelakowska K, Kryś J. The impact of COVID-19 on healthcare workers' absenteeism: infections, quarantines, sick leave - a database analysis of the Antoni Jurasz University Hospital No. 1. in Bydgoszcz Poland. Medical Research Journal. 2021; 6(1): 47-52, doi: 10.5603/mrj. a2021.0012.

9. Lackowski P, Piasecki M, Kasprzak M, et al. COVID-19 pandemic year in the cardiology department. Medical Research Journal. 2021; 6(1): 40-46, doi: 10.5603/mri.a2021.0009.

10. Grzelakowska K, Kasprzak M, Kryś J. COVID-19 and diabetes a deadly duo? Medical Research Journal. 2021; 6(2): 119-124, doi: 10.5603/mrj.2021.0030. 